\title{
Potential of Next-Generation Sequencing in Noninvasive Fetal Molecular Blood Group Genotyping
}

\author{
Sandra Wienzek-Lischka Sandy Bachmann Vanessa Froehner Gregor Bein \\ Institute for Clinical Immunology and Transfusion Medicine, Justus-Liebig University, Giessen, Germany
}

\section{Keywords}

Fetus · Newborn · Hemolytic disease · Alloimmune thrombocytopenia · Cell-free (fetal) DNA · Next-generation sequencing · Noninvasive fetal blood group genotyping

\begin{abstract}
Hemolytic disease of the fetus and newborn and fetal and neonatal alloimmune thrombocytopenia are caused by maternal antibodies against fetal alloantigens on red blood cells or platelets that are inherited from the father. After transplacental transport to the fetal circulation, antibodies of the lgG class may cause severe fetal anemia or bleeding complications. The indication for noninvasive fetal blood group genotyping is given if a clinically relevant antibody is detected in a pregnant woman and if the father is heterozygous (or unknown) for the implicated blood group allele. This mini-review will focus on the advantages and current limitations of next-generation sequencing (NGS) for noninvasive diagnosis of fetal blood groups which is, in contrast to fetal aneuploidy screening, proposed only by some research groups. Targeted massively parallel sequencing of short DNA fragments from maternal cell-free plasma samples enables counting of fetal alleles for many single nucleotide polymorphisms in parallel. This information can be utilized for estimation of the fetal fraction of cell-free DNA (cfDNA) as well as detection of the paternal blood group allele in question. Adherence to a cut-off of $\geq 4 \%$ fetal fraction for reporting conclusive results is recommended to avoid falsenegative results due to low fetal fraction. For screening purposes of fetal RHD in RhD-negative pregnant women, real-
\end{abstract}

time PCR methods are very well established. However, for diagnostic purposes, the targeted amplicon-based NGS approach has the inherent capability to estimate the fetal fraction of cfDNA. In the future, improving the accuracy of NGS by consensus sequencing of single cfDNA molecules may enable reliable fetal blood group genotyping already in the first trimester of pregnancy.

(c) 2020 S. Karger AG, Basel

\section{Hemolytic Disease of the Fetus and Newborn}

Hemolytic disease of the fetus and newborn (HDFN) is caused by maternal antibodies against fetal red blood cell (RBC) alloantigens inherited from the father. After transplacental transport to the fetal circulation, antibodies of IgG class may cause fetal anemia by opsonization and subsequent destruction of RBCs or by inhibition of fetal hematopoiesis, leading to fetal hydrops and death if untreated. Postnatally, destruction of RBCs of the neonate may cause severe hyperbilirubinemia, and if untreated, to precipitation of bilirubin in neuronal cells of basal ganglia, a condition named kernicterus, leading to death or severe neurologic disability in survivors. Maternal anti-D antibodies are associated with the highest risk of fetal morbidity and mortality [1]. The risk to develop severe HDFN is lower in pregnant women who are immunized against other RBC antigens compared to women who are immunized against $\mathrm{RhD}$ and $\mathrm{K}$. In a large prospective cohort study including 305,000 pregnancies, anti-K antibodies caused severe HDFN in $26 \%$ of the pregnancies at 
risk (antigen-positive fetus), anti-c antibodies in 10\%, anti-E antibodies in 2\%, and antibodies against other $\mathrm{Rh}$ antigens in 5\% of at-risk pregnancies [2]. Other RBC antibodies were not associated with severe HDFN in this study. Administration of anti-D immunoglobulin to $\mathrm{RhD}$-negative pregnant women prevents the immune response of the mother upon exposure to $\mathrm{RhD}$-positive $\mathrm{RBCs}$ of the fetus. The introduction of routine antenatal and postnatal anti-D immunoglobulin prophylaxis led to a reduction of the risk of severe HDFN caused by anti-D to 1 in 1,000 $\mathrm{RhD}$-negative pregnancies at risk [3]. Severe HDFN due to antibodies other than anti-D occurs in approximately 10 per 100,000 neonates [2]. In developed countries, routine RBC alloantibody screening in pregnant women has been implemented. Management of immunized pregnant women at risk includes screening for fetal anemia by Doppler measurement of peak systolic velocity in the fetal middle cerebral artery and by ultrasound examination of other signs of fetal anemia (cardiomegaly, ascites, hydrops). If fetal anemia is present, intravascular intrauterine transfusion of antigen-negative RBCs to the fetus is associated with favorable outcome in experienced referral centers for fetal therapy [4]. For further reading on the pathophysiology, diagnosis, treatment, and prevention of HDFN, we refer to a recent review [1].

\section{Fetal and Neonatal Alloimmune Thrombocytopenia}

Fetal and neonatal alloimmune thrombocytopenia (FNAIT) is caused by maternal antibodies against fetal platelet antigens which, after transplacental transport, may cause thrombocytopenia and bleeding complications in the fetus or newborn. Most of the FNAIT cases in Caucasians are caused by maternal antibodies against paternally inherited human platelet antigen (HPA)-1a [for review see 5]. The incidence of FNAIT is 1 in 1,000 live births, and the most severe consequence of FNAIT, intracranial hemorrhage ( $\mathrm{ICH}$ ) leading to death or neurologic disability, occurs in 10 per 100,000 neonates, commonly before birth [6]. Routine nationwide screening programs for the presence of anti-HPA antibodies in pregnant women [7] have not been established up to now. Without routine screening, interventions to reduce the incidence of ICH due to FNAIT are restricted to the management of subsequent pregnancies. A retrospective cohort study, including 43 cases of ICH due to FNAIT, assessed the gestational age at the onset of bleeding [8]. ICH often occurred during the second trimester and the clinical outcome was poor. Thus, strategies to prevent ICH caused by FNAIT will require the identification of at-risk pregnancies, i.e. an anti-HPA-immunized woman carrying a fetus with inheritance of the cognate antigen, early in the sec- ond trimester [8]. Current standard in the management of subsequent at-risk pregnancies is a noninvasive strategy including fetal bleeding prophylaxis with weekly maternal i.v. immunoglobulin ( $1 \mathrm{~g} / \mathrm{kg}$ body weight) starting at 12-16 weeks of gestation in high-risk pregnancies (ICH in a previous pregnancy) and at 20 weeks of gestation in standard risk pregnancies (no ICH in a previous pregnancy) [9]. For further reading on the pathophysiology, diagnosis, treatment, and prevention of FNAIT, we refer to a recent review [5].

\section{Cell-Free Fetal DNA in Plasma of Pregnant Women}

The discovery of fetal cell-free DNA (cfDNA) in plasma of pregnant women by Lo et al. [10] in 1997 rapidly led to the development of noninvasive prenatal testing for fetal RHD status $[11,12]$, fetal aneuploidy, and monogenic diseases [for review see 13].

\section{Origin and Properties of cfDNA}

cfDNA is released from dying cells (necrotic or apoptotic cell death) or actively released form living cells (exocytosis or NETosis) [14]. cfDNA represents small fragments of double-stranded DNA with nucleosome footprints, and hematopoietic cell death is the normal source of cfDNA [15]. A dominant population of cfDNA has a size of 166 bp representing the nucleosome core plus a linker, whereas placentally derived fetal DNA has a predominant size of $143 \mathrm{bp}$, representing the nucleosome core without a linker [16]. Certain genomic locations are overrepresented at the ends of plasma DNA fragments and these ends differ between maternal and fetal fragments [16]. A recent study on quantifying cfDNA in 104 healthy individuals estimated a median cfDNA plasma concentration of $1.64 \times 10^{3}$ copies $/ \mathrm{mL}$, corresponding to $5.43 \mathrm{ng} / \mathrm{mL}$ plasma with large interindividual differences [14] (the mean [male and female] weight of one human haploid genome is $3.23 \mathrm{pg}$ [17]). A finding of this study was that cfDNA median levels were 2- to 3-fold higher at 9:00 a.m. compared to later blood collection time points [14]. The authors recommend that fasting blood samples should be used when prenatal testing is considered. However, this finding must be validated with clinical samples of pregnant women. Lo et al. [18] showed that the mean fetal fraction of maternal cfDNA is $3.4 \%$ (range 0.39 $11.9 \%$ ) in early pregnancy and $6.2 \%$ (range $2.33-11.4 \%$ ) in late pregnancy, corresponding to 25.4 genome equivalents/mL (range 3.3-69.4) in early pregnancy and 292.2 genome equivalents/mL (range 76.9-769) in late pregnancy. Newer publications reported higher mean concentration of fetal cfDNA (cffDNA) of 14\% (range less 
than 4-30\%) [19]. Currently, many laboratories offering noninvasive fetal testing utilize a cut-off of $\geq 4 \%$ fetal fraction [20]. When fetal fraction values are lower than this cut-off, an inconclusive result is reported. Provided, plasma of a pregnant woman contains 1,000 copies cfDNA/ $\mathrm{mL}$, a fetal fraction of $4 \%$ corresponds to 40 copies $/ \mathrm{mL}$ of fetal DNA, and this relates to 20 copies/mL of paternally inherited alleles. Thus, noninvasive prenatal diagnosis (NIPD) of fetal blood groups must face two challenges: first, a low copy number of paternal alleles in maternal plasma, particularly in early pregnancy, and second, short fetal DNA fragments. In consequence, amplicon-based methods for the detection of paternal alleles in maternal plasma must target small size sequences to reach high sensitivity.

\section{Noninvasive Prenatal Screening for Fetal RHD Status in RhD-Negative Women}

In a growing number of countries, all nonsensitized $\mathrm{RhD}$-negative pregnant women receive antenatal anti- $\mathrm{D}$ immunoglobulin prophylaxis without knowledge of the fetal RHD status. Noninvasive prenatal testing of cell-free fetal DNA in maternal plasma could avoid unnecessary anti-D immunoglobulin administration in up to $40 \%$ of $\mathrm{RhD}$-negative pregnant women $[21,22]$. Noninvasive fetal RHD typing to guide anti-D immunoglobulin prophylaxis has been implemented in a nationwide program in several European countries [for review see 21, 22], and the high sensitivity [23] of the prenatal testing justified the abolition of cord blood serology to guide postnatal anti-D immunoglobulin prophylaxis [22]. All national screening programs are based on real-time PCR assays and do not include positive controls for the presence of fetal DNA [22].

\section{Diagnostic Fetal Blood Group Genotyping}

Diagnostic measures and prophylactic or therapeutic interventions in pregnant immunized women are only indicated if fetal RBCs or platelets carry the cognate alloantigen against which the maternal alloantibody is directed. If the father is heterozygous for the implicated alloantigen, the likelihood that the fetus will inherit the paternal allele is $50 \%$. In this case, the pregnancy is at risk and follow-up is indicated. Thus, the indication for diagnostic fetal blood group genotyping is given if a clinically relevant alloantibody is detected in the mother and if the father is heterozygous (or unknown) for the implicated blood group allele. If the father is homozygous, fetal blood group genotyping is not indicated unless the paternity is disputed. Fetal blood group genotypes can be determined by chorionic villous sampling, amniocentesis, or cord blood sampling. Current studies demonstrated that the miscarriage rate at large experienced centers is 1 in 500 for chorionic villous sampling and 1 in 1,000 for amniocentesis [24]. Furthermore, invasive procedures are associated with the risk of de novo or a boosted humoral immune response in the mother against fetal blood group alloantigens [25]. Noninvasive fetal genotyping for K, Rh C, c, E, and HPA-1a, mainly by real-time PCR with and without prior digestion of maternally derived alleles with restriction enzyme has been described [for review see 22]. These methods do not have internal controls for the presence of fetal DNA and may lead to false-negative results in cases where the fraction of cffDNA in maternal plasma is below the detection limit. External positive controls for fetal DNA include the detection of the male SRY gene, genetic variation that differs between the mother and the fetus, and epigenetic variation, for example hypermethylation of the placental RASSF1A gene [26]. Because of the potentially severe consequences of false-negative results in alloimmunized women, a blood group antigen-negative result should only be issued if fetal DNA is demonstrated [27]. However, screening programs for fetal RHD determination as established in Denmark, Finland, and the Netherlands reported high accuracy due to the detection of presence or absence of $R H D$ exon sequences [28]. Detection of other blood group systems, especially fetal $\mathrm{K}$, which differ only in one single nucleotide is more sophisticated [22].

The genetic basis of the most relevant $\mathrm{RBC}$ alloantigens and platelet alloantigens in HDFN and FNAIT has been reviewed recently by van der Schoot et al. [22]. This mini-review will therefore focus on the advantages and limitations of next-generation sequencing (NGS) for noninvasive diagnosis of fetal blood groups.

\section{Rationale for Use of NGS in Diagnostic Fetal Blood Group Genotyping}

Many noninvasive fetal aneuploidy-screening systems are based on NGS. For the purpose of fetal blood group genotyping, only a few publications exist [29-31].

The key steps of NGS technologies are (1) clonal in vitro amplification of DNA fragments and (2) multiplexing millions to billions of immobilized templates. The clonal PCR amplification of single molecules takes place, after limiting dilution, either in an emulsion of beads (IonTorrent platform) [32] or by "bridge amplification" of single molecules immobilized on a surface (Illumina platform). Sequencing by polymerase-mediated incorporation of nucleotides comprises detection of ion release on a microchip array, containing one bead per microwell (IonTorrent platform) or incorporation of reversibly termi-
Wienzek-Lischka/Bachmann/Froehner/ Bein 


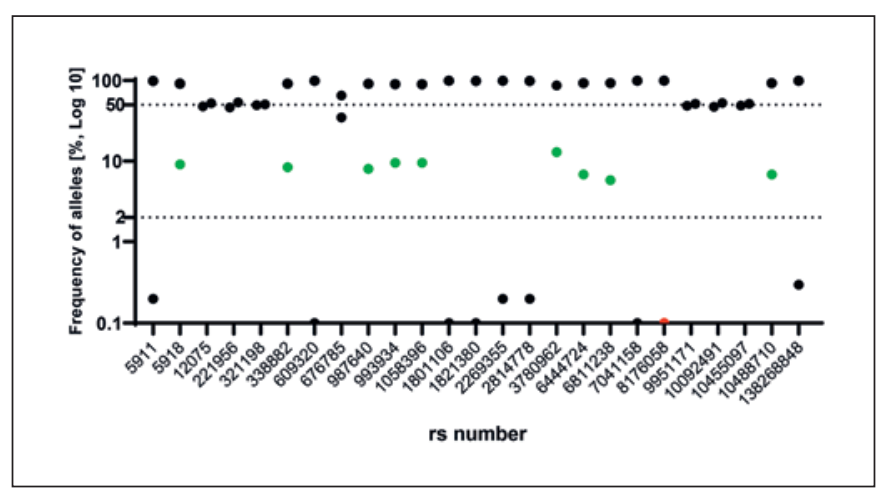

Fig. 1. Example of noninvasive fetal blood group genotyping by targeted next-generation sequencing of cfDNA. Target sequences flanking multiple single nucleotide polymorphisms (SNPs; rs numbers are shown on the $x$ axis) were amplified from cfDNA of a pregnant woman (31 weeks of gestation) with anti-K antibody and clonally sequenced (Illumina platform). Allele frequencies of SNPs are plotted for each target sequence (Y chromosome and $R H D$-specific reads not shown). Dotted line at $2 \%$ : predefined cutoff for paternally inherited alleles ( $4 \%$ fetal fraction). Allele frequencies clustering at 50\%: maternal heterozygosity. Green data points: paternally inherited fetal alleles. Red data point: frequency of reads representing the $\mathrm{K}$ allele. A K-negative phenotype of the fetus is predicted.

nating, reversibly fluorescent dNTPs to the templates immobilized on a surface, and optical readout to determine which of four colors was incorporated by each template (Illumina platform). We do not review other NGS methods, e.g. nanopore sequencing, and refer for further reading to a recent review [33]. Rieneck et al. [29] were the first to propose NGS for antenatal prediction of fetal blood group phenotype $(\mathrm{K})$ from cfDNA. This approach did not include quantification of the fetal fraction. Wienzek-Lischka et al. [30] were the first to propose a comprehensive NGS method that included sequencing of all relevant blood group polymorphisms as well as irrelevant/ anonymous single-nucleotide polymorphisms (SNPs) for estimation of the fetal fraction. A similar approach was published recently by Orzinska et al. [31]. Nevertheless, these publications described a small number of tested samples and should be considered as "proof-of-concept" studies.

\section{NGS Sequencers as an Allele-Counting Device and Estimation of Fetal Fraction}

For the use of NGS in aneuploidy screening, two main sequencing approaches for the analysis of cfDNA exist: random (whole-genome) and targeted sequencing. In the whole-genome sequencing approach, maternal and fetal cfDNA molecules are randomly sequenced, and

Noninvasive Fetal Blood Group

Genotyping mapped to specific chromosomes. The numbers of DNA molecules mapped to different human chromosomes are then counted. For pregnancies expecting a fetus with trisomy 21 , the proportion of $\mathrm{cfDNA}$ molecules derived from chromosome 21 is probably higher than that in a reference data set based on samples from pregnant women carrying euploid fetuses. In the targeted method, SNPs on the chromosomes of interest are amplified and sequenced. Ratios between heterozygous SNP alleles are compared with those of other targeted chromosomes [13]. To our knowledge, blood group information cannot be extracted from fetal anomaly screening in currently available tests.

For the use of fetal blood group typing, cfDNA isolation from maternal plasma and targeted clonal amplification of short DNA sequences flanking known single nucleotide polymorphisms (SNPs), and subsequent multiplex sequencing allow for "allele counting" (since the original templates were amplified, the counts represent relative numbers) of many different SNPs in parallel. On the one hand, fetal SNPs coding for all relevant blood groups can be detected in parallel [30]. On the other hand, inclusion of irrelevant/anonymous SNPs, ideally with an average heterozygosity of 0.5 , irrespective of ethnicity [34], can be used to estimate the fetal fraction if the mother is homozygous for one of the alleles and the fetus inherited the antithetical allele from his father [30] (see also Fig. 1).

\section{NGS for Haplotype Phasing}

Sequences generated by NGS represent the sequence of diluted single molecules that are clonally sequenced and thus enable phasing of short haplotypes. The advantage of single molecule sequencing for noninvasive fetal blood group genotyping may be illustrated by diagnostic fetal $R H C E^{*}$ e genotyping. The $R H C E^{*}$ E/e polymorphism is caused by a $\mathrm{C}>\mathrm{G}$ nucleotide exchange at position c.676 in exon 5 of the RHCE gene, where $\mathrm{G}$ is specific for the Rh e antigen. The c.676G residue is also present in the $R H D$ gene. Thus, interrogating c.676, e.g. by real-time PCR, would not allow for identification of fetal $R H C E$-specific sequence of the e allele, since the same nucleotide occurs in $R H D$. However, $R H C E$-specific reads can be identified by adjacent SNP (c.667C) on the same haplotype enabling

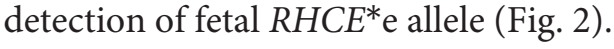

\section{Technical Considerations}

\section{Preanalytics}

Laboratory testing can be divided into different phases: the preanalytical, analytical, and postanalytical phases. 


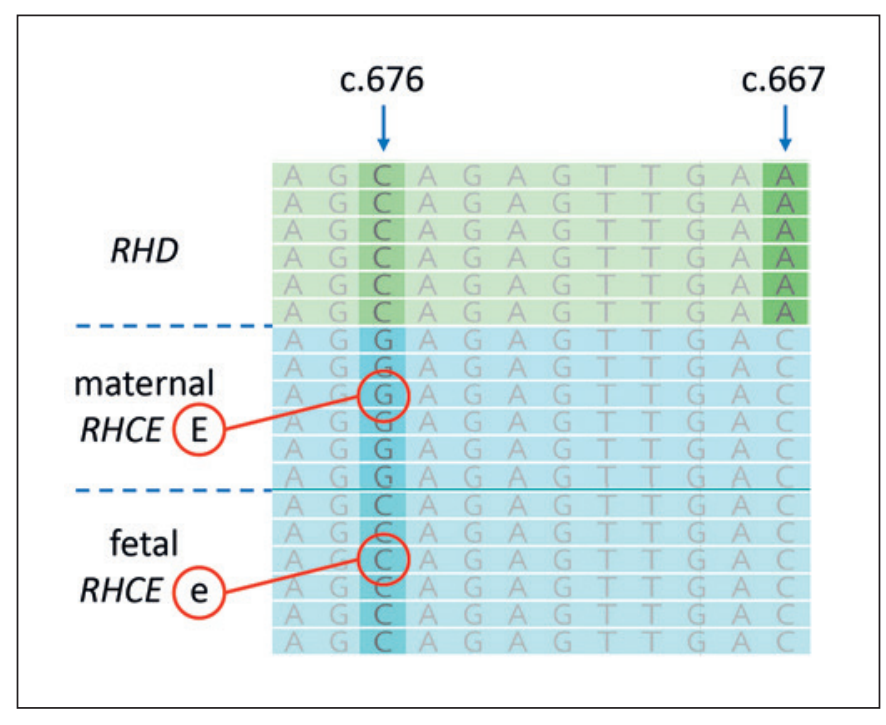

Fig. 2. Identification of fetal $R H C E^{*}$ e allele by haplotype phasing. Target sequence surrounding position c.676 of homologous $R H D$ and RHCE genes was amplified from maternal cfDNA and clonally sequenced (Illumina platform). Sequence reads were mapped to reference and sorted by position c.676 and position c.667 employing Geneious Prime (Biomatters Ltd., Auckland, New Zealand). The figure provides a graphical view of sequence alignments (complementary bases in reverse direction compared to the reference transcript coding sequences LRG_796 (RHD) and LRG_797 (RHCE); locus reference genomic; http://www.lrg-sequence.org). The fetal $R H C E^{*}$ e allele (c.676C; c.676G in the reference sequence of $R H C E^{*} \mathrm{e}$ ) can be identified by sorting RHCE haplotypes according to $R H C E$-specific sequence at position c.667. Interrogating c.676 alone, e.g. by real-time PCR, would not allow for identification of fetal RHCE-specific sequence of the RHCE*e allele, since the same nucleotide occurs in $R H D$.

In the preanalytical phase, identification and selection of an appropriate test, specimen collection (e.g., wrong sample tubes, hemolysis, or insufficient volume) and transport conditions (e.g., temperature, duration) but also aliquoting mistakes are important. Laboratory testing is performed in the analytical phase. The postanalytical phase comprises analysis of data, interpretation of results, and reporting. During this process, $46-68 \%$ of errors occur in the preanalytical phase, which adversely affects the quality of the data in the following phases [35]. Knowledge of preanalytics and cfDNA extraction was mainly obtained from high-throughput NIPD (aneuploidy screening, fetal RHD screening).

For NIPD, an adequate amount of cffDNA must be present to obtain a reliable test result. Proper sample collection and avoidance of degradation of white blood cells of maternal origin are important to preserve the fetal fraction and keep a low amount of maternal cfDNA in the sample. For NIPD, dedicated blood collection tubes (e.g., Cell-Free DNA BCT, Streck) are recommended that stabilize the sample and prevent degradation of maternal leukocytes and release of genomic DNA [36]. Novel approaches to stabilize cfDNA are formaldehyde free blood collection tubes which are able to inhibit the blood cell metabolism in blood samples [37].

In comparison to EDTA blood, the cfDNA/cffDNA ratio remains stable up to 5 days at room temperature. After blood withdrawal, these dedicated collection tubes (e.g., Cell-Free DNA BCT, Streck) must be inverted gently several times to mix the preserving agent. The tubes must not be refrigerated, frozen, or opened until processed. Half-filled tubes due to an incomplete sample draw are not acceptable. Hemolytic samples are not acceptable for NIPD because free hemoglobin may inhibit PCR reaction. EDTA-anticoagulated samples are sufficient for non-NGS based fetal RHD screening methods [38]. Newer data recommend an immediate processing after phlebotomy (up to 2 days) [39] and showed that the fragment size in EDTA plasma samples is longer compared to fragments extracted from dedicated tubes, possibly due to DNA release from degrading leukocytes [40]. These samples should be centrifuged as soon as possible within $8 \mathrm{~h}$, and plasma should be separated and refrigerated $\left(<-20^{\circ} \mathrm{C}\right)[40,41]$.

Serum samples for extraction of cfDNA are used particularly in retrospective studies using bio-banked samples. There are differences between plasma- and serumderived cfDNA. Serum contains 2- to 24-fold higher cfDNA concentrations compared to plasma but lower fetal fractions [42].

Beside specimen collection and transport conditions, other factors can decrease the fetal fraction, which can cause analytical failures or false-negative results. Prior to 10 weeks of gestation, the cffDNA amount is relatively low and increases slowly ( $0.1 \%$ per week) with gestational age from 10 to approximately 20 weeks of gestation. In the third trimester of pregnancy, fetal fraction increases approximately $1 \%$ per week. Fetal fraction decreases with increasing maternal weight $[39,43]$. This inverse relationship is caused by dilution of a relatively constant amount of cffDNA in a larger maternal plasma volume of obese women and to an increased concentration of maternally derived cfDNA as maternal weight increases. In trisomy 18, the fetal fraction is lower than in euploid pregnancies. In vitro fertilization [44] and twin pregnancy are also associated with lower amounts of cffDNA [45]. The fetal fraction in maternal plasma cfDNA increases with serum pregnancy-associated plasma protein- $A$ and free human chorionic gonadotropin ( $\beta$-hCG). No effects on fetal fraction have been attributed to maternal age, racial origin, smoking status, fetal gender, and trisomy 21 karyotype [46]. The use of low-molecular-weight heparin has no effect on cffDNA fraction as demonstrated in recent studies [47], in contrast to earlier reports [48]. Autoimmune disorders like SLE or APL are often treated with 


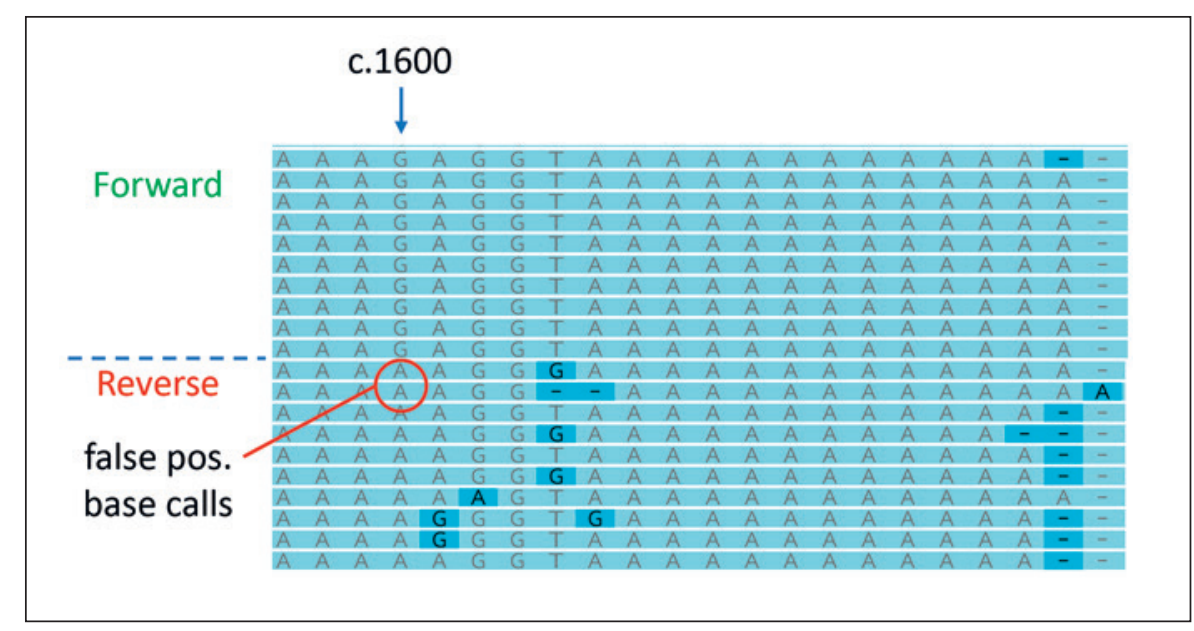

Fig. 3. Sequencing errors adjacent to homopolymer-containing regions. Target sequence surrounding position c. 1600 of ITGA2 was amplified from maternal cfDNA and clonally sequenced (Illumina platform). The HPA-5a/b polymorphism is caused by a G>A nucleotide exchange at this position, where $\mathrm{G}$ is specific for the HPA$5 \mathrm{a}$ antigen. Sequence reads were mapped to reference and sorted by position c. 1600 and read direction (forward and reverse) em-

low-molecular-weight heparin and could affect cfDNA results either by distorting cfDNA measurement or by impacting the fetal fraction. Many authors have reported increased levels of cfDNA in patients with active SLE which could lead to a low fetal fraction [47].

\section{cfDNA Extraction}

The choice of DNA extraction method is a critical step. A variety of commercial kits for isolation of circulating nucleic acids are available. Column-based or magnetic bead extractions methods are the common methods used. Cellfree total DNA yield and resulting cffDNA fraction differ and also the fragment size of isolated DNA [49]. These parameters influence subsequent analysis and should be optimized. Recently, fetal placenta-specific epigenetic regions (FSERs) have been identified for quantification of cffDNA for noninvasive prenatal testing. Lim et al. [50] compared column-based and magnetic bead-based methods. The quantity of methylated FSERs was significantly higher for extracted DNA using the column-based kit than that extracted using the magnetic bead-based kit $(p<0.001$ for all FSERs). Akbariqomi et al. [51] compared commercially available kits with the triton/heat/phenol/glycogen (THPG) and conventional triton/heat/phenol methods. The genome-equivalent $/ \mathrm{mL}$ values of methylated cffDNA extracted using the THPG method were significantly higher compared to the other extraction methods.

\section{Assay Design}

For fetal blood group genotyping, an amplicon-based targeted sequencing approach is recommended [30]. The ploying Geneious Prime (Biomatters Ltd., Auckland, New Zealand). The figure provides a graphical view of sequence alignments to the reference transcript coding sequence LRG_1001 (ITGA2) (locus reference genomic; http://www.lrg-sequence.org). Sequence reads in reverse direction lead to false-positive base calls of the HPA-5b allele ahead of a homopolymer sequence of adenosines.

short fragment size of cffDNA should be regarded in the primer design. Sequencing short cfDNA fragments is a reasonable strategy to reduce the probability of no call results because of low fetal fraction [52, 53]. For NIPD of fetal blood groups, we performed library preparation according to the manufacturer's instruction (Ion AmpliSeq Library Kit 2.0, Life Technologies/Thermo Fisher Scientific, Carlsbad, CA, USA, or Ion AmpliSeq Library plus kit for Illumina, San Diego, CA, USA) using a custommade primer panel (Ion AmpliSeq Designer). The targeted sequences were chosen to flank SNPs/exonic regions encoding common blood group systems of platelets and RBCs that are frequently involved in FNAIT or HDFN: ITGB3 (HPA-1), ITGA2B (HPA-3), ITGA2 (HPA-5), CD109 (HPA-15), RHD, RHCE, KEL, DARC, SLC14A1, GYPB, and Y-chromosome located target sequences [30]. For estimation of fetal fraction, AmpliSeq Sample ID-Panel (Thermo Fisher Scientific, Carlsbad, CA, USA) targeting eight SNPs that are unlinked, nonexonic markers that show consistently high minor allele frequency across a diverse group of human populations and additional 6 SNP targets [34] were included. Depending on library preparation, sequencing was done on Ion Torrent Personal Genome Machine (PGM; Thermo Fisher Scientific) or MiSeq (Illumina) platforms.

\section{Fetal Allele Calling}

If the sequence quality parameters of raw data met the acceptance criteria, data analysis was performed. Unsuccessful amplification was defined as total reads below 1,000 for a targeted sequence. Unexpected base calls were 
defined as all base calls at an SNP position not belonging to the known biallelic system (e.g., in a biallelic system with $\mathrm{T}>\mathrm{C}$ polymorphism, all $\mathrm{A}$ and $\mathrm{G}$ calls were called "unexpected base calls"). If a pregnant woman was homozygous for a given SNP and the antithetical allele at that locus was detected with a frequency of $\geq 2 \%$ of total reads ( $\geq 4 \%$ fractional fetal DNA), this allele was considered a paternally inherited fetal allele. An example of noninvasive fetal blood group genotyping is shown in Figure 1.

\section{Limitations}

Currently, in our experience of sequencing more than 200 patient samples [unpubl.], there are two limitations in NIPD of fetal blood groups. First, the introduction of sequencing errors (unexpected base calls) either by PCR amplification or sequencing artifacts limits the sensitivity. Especially, sequencing errors might occur when sequencing homopolymer regions (Fig. 3). In our hands, the mean rate of false-positive base calls across all targeted SNPs is $0.15 \%$. If we calculate the limit of detection (LOD) by the equation mean of blank $+3 *$ standard deviation of the blank, the current LOD would be $0.43 \%$, and the limit of quantification (mean of blank $+10 *$ standard deviation) would be $1.1 \%$ [unpubl.]. Thus, we recommend adherence to the cut-off of $\geq 4 \%$ fetal fraction ( $\geq 2 \%$ paternal al- leles) for reporting conclusive results. Second, allelic dropout leading to a false-negative result, even in cases with fetal fraction $\geq 4 \%$, cannot totally be excluded. Therefore, if the result is negative, we recommend confirmatory testing after 20 weeks' gestation. Adhering to these recommendations, we did not encounter false-negative or falsepositive results during the last 3 years [unpubl.].

\section{Conclusion}

From our experience, targeted NGS has the potential to be a reliable method for diagnostic noninvasive fetal blood group genotyping due to the inherent capability to estimate the fetal fraction of cfDNA. Improving the accuracy of NGS by consensus sequencing of single DNA molecules [54] may enable reliable fetal blood group genotyping already in the first trimester of pregnancy.

\section{Acknowledgement}

We thank Marion Ernst-Schlegel, Annika Krautwurst, and Marion Soelter for excellent technical assistance.

\section{Disclosure Statement}

The authors declared no conflict of interest.

\section{References}

1 de Haas M, Thurik FF, Koelewijn JM, van der Schoot CE. Haemolytic disease of the fetus and newborn. Vox Sang. 2015 Aug;109(2): 99-113.

2 Koelewijn JM, Vrijkotte TG, van der Schoot CE, Bonsel GJ, de Haas M. Effect of screening for red cell antibodies, other than anti-D, to detect hemolytic disease of the fetus and newborn: a population study in the Netherlands. Transfusion. 2008 May;48(5):941-52.

3 Koelewijn JM, de Haas M, Vrijkotte TG, Bonsel GJ, van der Schoot CE. One single dose of 200 microg of antenatal RhIG halves the risk of anti-D immunization and hemolytic disease of the fetus and newborn in the next pregnancy. Transfusion. 2008 Aug;48(8): 1721-9.

4 Zwiers C, Oepkes D, Lopriore E, Klumper FJ, de Haas M, van Kamp IL. The near disappearance of fetal hydrops in relation to current state-of-the-art management of red cell alloimmunization. Prenat Diagn. 2018 Nov; 38(12):943-50.

5 Winkelhorst D, Oepkes D. Foetal and neonatal alloimmune thrombocytopenia. Best Pract Res Clin Obstet Gynaecol. 2019 Jul;58:15-27.

6 Kamphuis MM, Paridaans NP, Porcelijn L, Lopriore E, Oepkes D. Incidence and consequences of neonatal alloimmune thrombocytopenia: a systematic review. Pediatrics. 2014 Apr;133(4):715-21.
7 Kjeldsen-Kragh J, Killie MK, Tomter G, Golebiowska E, Randen I, Hauge R, et al. A screening and intervention program aimed to reduce mortality and serious morbidity associated with severe neonatal alloimmune thrombocytopenia. Blood. 2007 Aug;110(3):833-9.

8 Tiller H, Kamphuis MM, Flodmark O, Papadogiannakis N, David AL, Sainio S, et al. Fetal intracranial haemorrhages caused by fetal and neonatal alloimmune thrombocytopenia: an observational cohort study of 43 cases from an international multicentre registry. BMJ Open. 2013 Mar;3(3):3.

9 Winkelhorst D, Murphy MF, Greinacher A, Shehata N, Bakchoul T, Massey E, et al. Antenatal management in fetal and neonatal alloimmune thrombocytopenia: a systematic review. Blood. 2017 Mar;129(11):1538-47.

10 Lo YM, Corbetta N, Chamberlain PF, Rai V, Sargent IL, Redman CW, et al. Presence of fetal DNA in maternal plasma and serum. Lancet. 1997 Aug;350(9076):485-7.

11 Faas BH, Beuling EA, Christiaens GC, von dem Borne AE, van der Schoot CE. Detection of fetal RHD-specific sequences in maternal plasma. Lancet. 1998 Oct;352(9135):1196.

12 Lo YM, Hjelm NM, Fidler C, Sargent IL, Murphy MF, Chamberlain PF, et al. Prenatal diagnosis of fetal RhD status by molecular analysis of maternal plasma. N Engl J Med. 1998 Dec; 339(24):1734-8.
13 Bianchi DW, Chiu RW. Sequencing of Circulating Cell-free DNA during Pregnancy. N Engl J Med. 2018 Aug;379(5):464-73.

14 Meddeb R, Dache ZA, Thezenas S, Otandault A, Tanos R, Pastor B, et al. Quantifying circulating cell-free DNA in humans. Sci Rep. 2019 Mar;9(1):5220.

15 Snyder MW, Kircher M, Hill AJ, Daza RM, Shendure J. Cell-free DNA Comprises an In Vivo Nucleosome Footprint that Informs Its Tissues-Of-Origin. Cell. 2016 Jan;164(1-2): 57-68.

16 Chan KC, Jiang P, Sun K, Cheng YK, Tong $\mathrm{YK}$, Cheng $\mathrm{SH}$, et al. Second generation noninvasive fetal genome analysis reveals de novo mutations, single-base parental inheritance, and preferred DNA ends. Proc Natl Acad Sci USA. 2016 Dec;113(50):E8159-68.

17 Piovesan A, Pelleri MC, Antonaros F, Strippoli P, Caracausi M, Vitale L. On the length, weight and GC content of the human genome. BMC Res Notes. 2019 Feb;12(1):106.

18 Lo YM, Tein MS, Lau TK, Haines CJ, Leung TN, Poon PM, et al. Quantitative analysis of fetal DNA in maternal plasma and serum: implications for noninvasive prenatal diagnosis. Am J Hum Genet. 1998 Apr;62(4):768-75. 
19 Barrett AN, Xiong L, Tan TZ, Advani HV, Hua R, Laureano-Asibal C, et al. Measurement of fetal fraction in cell-free DNA from maternal plasma using a panel of insertion/ deletion polymorphisms. PLoS One. 2017 Oct;12(10):e0186771.

20 Benn P, Cuckle H. Theoretical performance of non-invasive prenatal testing for chromosome imbalances using counting of cell-free DNA fragments in maternal plasma. Prenat Diagn. 2014 Aug;34(8):778-83.

21 Finning K, Martin P, Summers J, Massey E, Poole G, Daniels G. Effect of high throughput RHD typing of fetal DNA in maternal plasma on use of anti-RhD immunoglobulin in RhD negative pregnant women: prospective feasibility study. BMJ. 2008 Apr;336(7648):816-8

22 van der Schoot CE, Winkelhorst D, Clausen FB. Noninvasive Fetal Blood Group Typing. In: Klein HG, Page-Christiaens L, editors: Noninvasive prenatal testing (NIPT): Applied genomics in prenatal screening and diagnosis. London: Academic Press; 2018. p. 125-156.

23 Clausen FB, Barrett AN, Akkök CA, Armstrong-Fisher S, Bergström KD, Boggione CT, et al.; Noninvasive Fetal RHD Genotyping EQA2017 Working Group. Noninvasive fetal RHD genotyping to guide targeted anti-D prophylaxis-an external quality assessment workshop. Vox Sang. 2019 May;114(4):386-93.

24 Kozlowski P, Burkhardt T, Gembruch U, Gonser M, Kähler C, Kagan KO, et al. DEGUM, ÖGUM, SGUM and FMF Germany Recommendations for the Implementation of First-Trimester Screening, Detailed Ultrasound, Cell-Free DNA Screening and Diagnostic Procedures. Ultraschall Med. 2019 Apr;40(2):176-93.

25 Kähler C, Gembruch U, Heling KS, Henrich W, Schramm T; DEGUM. Empfehlungen der DEGUM zur Durchführung von Amniozentese und Chorionzottenbiopsie. Ultraschall Med. 2013 Oct;34(5):435-40.

26 Chan KC, Ding C, Gerovassili A, Yeung SW, Chiu RW, Leung TN, et al. Hypermethylated RASSF1A in maternal plasma: A universal fetal DNA marker that improves the reliability of noninvasive prenatal diagnosis. Clin Chem. 2006 Dec;52(12):2211-8.

27 Scheffer PG, de Haas M, van der Schoot CE. The controversy about controls for fetal blood group genotyping by cell-free fetal DNA in maternal plasma. Curr Opin Hematol. 2011 Nov;18(6):467-73

28 Yang H, Llewellyn A, Walker R, Harden M, Saramago P, Griffin S, et al. High-throughput, non-invasive prenatal testing for fetal rhesus $\mathrm{D}$ status in RhD-negative women: a systematic review and meta-analysis. BMC Med. 2019 Feb;17(1):37.

29 Rieneck K, Bak M, Jønson L, Clausen FB, Krog GR, Tommerup N, et al. Next-generation sequencing: proof of concept for antenatal prediction of the fetal Kell blood group phenotype from cell-free fetal DNA in maternal plasma. Transfusion. 2013 Nov;53(11 Suppl 2):2892-8.
30 Wienzek-Lischka S, Krautwurst A, Fröhner V, Hackstein H, Gattenlöhner S, Bräuninger A, et al. Noninvasive fetal genotyping of human platelet antigen-1a using targeted massively parallel sequencing. Transfusion. 2015 Jun;55(6 Pt 2):1538-44.

31 Orzińska A, Guz K, Mikula M, Kluska A, Balabas A, Ostrowski J, et al. Prediction of fetal blood group and platelet antigens from maternal plasma using next-generation sequencing. Transfusion. 2019 Mar;59(3):1102-7.

32 Rothberg JM, Hinz W, Rearick TM, Schultz J, Mileski W, Davey M, et al. An integrated semiconductor device enabling non-optical genome sequencing. Nature. 2011 Jul; 475(7356):348-52

33 Shendure J, Balasubramanian S, Church GM, Gilbert W, Rogers J, Schloss JA, et al. DNA sequencing at 40: past, present and future. Nature. 2017 Oct;550(7676):345-53.

34 Pakstis AJ, Speed WC, Fang R, Hyland FC, Furtado MR, Kidd JR, et al. SNPs for a universal individual identification panel. Hum Genet. 2010 Mar;127(3):315-24.

35 Neumann MH, Bender S, Krahn T, Schlange T. ctDNA and CTCs in Liquid Biopsy - Current Status and Where We Need to Progress. Comput Struct Biotechnol J. 2018 Jun;16: 190-5.

36 Norton SE, Lechner JM, Williams T, Fernando MR. A stabilizing reagent prevents cellfree DNA contamination by cellular DNA in plasma during blood sample storage and shipping as determined by digital PCR. Clin Biochem. 2013 Oct;46(15):1561-5.

37 Fernando MR, Jiang C, Krzyzanowski GD, Somer-Shely T, Ryan WL. A novel approach to stabilize fetal cell-free DNA fraction in maternal blood samples for extended period of time. PLoS One. 2018 Dec;13(12):e0208508.

38 Müller SP, Bartels I, Stein W, Emons G, Gutensohn K, Legler TJ. Cell-free fetal DNA in specimen from pregnant women is stable up to 5 days. Prenat Diagn. 2011 Dec;31(13): $1300-4$.

39 Wang Q, Cai Y, Brady P, Vermeesch JR. Realtime PCR evaluation of cell-free DNA subjected to various storage and shipping conditions. Genet Mol Res. 2015 Oct;14(4):12797-804.

40 Mehrotra M, Singh RR, Chen W, Huang RS, Almohammedsalim AA, Barkoh BA, et al. Study of Preanalytic and Analytic Variables for Clinical Next-Generation Sequencing of Circulating Cell-Free Nucleic Acid. J Mol Diagn. $2017 \mathrm{Jul} ; 19(4): 514-24$.

41 Barrett AN, Zimmermann BG, Wang D, Holloway A, Chitty LS. Implementing prenatal diagnosis based on cell-free fetal DNA: accurate identification of factors affecting fetal DNA yield. PLoS One. 2011;6(10):e25202.

42 Wong FC, Sun K, Jiang P, Cheng YK, Chan $\mathrm{KC}$, Leung TY, et al. Cell-free DNA in maternal plasma and serum: A comparison of quantity, quality and tissue origin using genomic and epigenomic approaches. Clin Biochem. 2016 Dec;49(18):1379-86.
43 Livergood MC, LeChien KA, Trudell AS. Obesity and cell-free DNA "no calls": is there an optimal gestational age at time of sampling? Am J Obstet Gynecol. 2017 Apr;216(4): 413.e1-9.

44 Lee TJ, Rolnik DL, Menezes MA, McLennan AC, da Silva Costa F. Cell-free fetal DNA testing in singleton IVF conceptions. Hum Reprod. 2018 Apr;33(4):572-8.

45 del Mar Gil M, Quezada MS, Bregant B, Syngelaki A, Nicolaides KH. Cell-free DNA analysis for trisomy risk assessment in first-trimester twin pregnancies. Fetal Diagn Ther. 2014;35(3):204-11.

46 Ashoor G, Poon L, Syngelaki A, Mosimann B, Nicolaides KH. Fetal fraction in maternal plasma cell-free DNA at 11-13 weeks' gestation: effect of maternal and fetal factors. Fetal Diagn Ther. 2012;31(4):237-43.

47 Dabi Y, Guterman S, Jani JC, Letourneau A Demain A, Kleinfinger P, et al. Autoimmune disorders but not heparin are associated with cell-free fetal DNA test failure. J Transl Med. 2018 Dec;16(1):335.

48 Burns W, Koelper N, Barberio A, DeagostinoKelly M, Mennuti M, Sammel MD, et al. The association between anticoagulation therapy, maternal characteristics, and a failed cfDNA test due to a low fetal fraction. Prenat Diagn. 2017 Nov;37(11):1125-9.

49 Cook L, Starr K, Boonyaratanakornkit J, Hayden R, Sam SS, Caliendo AM. Does Size Matter? Comparison of Extraction Yields for Different-Sized DNA Fragments by Seven Different Routine and Four New Circulating Cell-Free Extraction Methods. J Clin Microbiol. 2018 Nov;56(12):1-13.

50 Lim JH, Lee BY, Kim JW, Han YJ, Chung JH, $\mathrm{Kim} \mathrm{MH}$, et al. Evaluation of extraction methods for methylated cell-free fetal DNA from maternal plasma. J Assist Reprod Genet. 2018 Apr;35(4):637-41.

51 Akbariqomi M, Heidari R, Gargari SS, Omrani $\mathrm{MD}$, Rigi G, Sanikhani NS, et al. Evaluation and statistical optimization of a method for methylated cell-free fetal DNA extraction from maternal plasma. J Assist Reprod Genet. 2019 May;36(5):1029-38.

52 Qiao L, Yu B, Liang Y, Zhang C, Wu X, Xue $\mathrm{Y}$, et al. Sequencing shorter cfDNA fragments improves the fetal DNA fraction in noninvasive prenatal testing. Am J Obstet Gynecol. 2019 Oct;221(4):345.e1-11.

53 Qiao L, Zhang Q, Liang Y, Gao A, Ding Y, Zhao $\mathrm{N}$, et al. Sequencing of short cfDNA fragments in NIPT improves fetal fraction with higher maternal BMI and early gestational age. Am J Transl Res. 2019 Jul;11(7): 4450-9.

54 Salk JJ, Schmitt MW, Loeb LA. Enhancing the accuracy of next-generation sequencing for detecting rare and subclonal mutations. Nat Rev Genet. 2018 May;19(5):269-85. 